\title{
Analysis of Clinicopathological Parameters and Ki 67 Immunoexpression in Urothelial Neoplasms of Bladder
}

\author{
Megala Chandrasekar ${ }^{1 *}$, Saranya $V^{2}$ and Surekha $B^{3}$ \\ 'Pathology, Vinayaka Missions Kirupananda Variyar Medical College.Salem, Tamil Nadu, INDIA. \\ ${ }^{2}$ SKS Hospitals, Salem. Tamil Nadu, INDIA \\ ${ }^{3}$ Vinayaka Missions Medical College, Karaikal-609609. India
}

\begin{abstract}
Background: Mitotic count is one of the most important determinant of proliferative capacity of any tumour. Immunohistochemical expression of ki 67 is an alternative and effective way of determining the growth fraction. This study was conducted to assess the expression of Ki 67 proliferative marker in bladder urothelial tumors, and to correlate proliferative markers with histopathological grading \& staging, thereby helps in identifying high risk patients for disease progression and recurrence who may thus benefit from perioperative systemic chemotherapy.

Methods: This study was conducted in department of pathology in collaboration with department of urology in the period of I year from March 2018-March 2019. Results was evaluated on paraffin embedded tissues of bladder neoplasm specimens by immunohistochemical methods and correlated with clinical parameters.

Result: In present study, high grade tumours have over expression of Ki 67 and graded as $3+$ and $4+$. Low grade tumours have low expression and have grades $1+$ and $2+$. Out of 30 cases, $40 \%$ (12 cases) show $4+$ and $27 \%$ show ( 8 cases) $3+$, both being high grade tumors. Whereas, $27 \%$ ( 8 cases) have $1+$ and $7 \%$ ( 2 cases) have $2+$, both being low grade tumours). p value is highly significant which is $\mathrm{p}<0.001$.

Conclusion: Expression of Ki 67 does correlate with grade and stage of the tumour thereby on prognostic significance. Hence ki 67 may be used as marker to predict aggressive behaviour of bladder neoplasms and also to differentiate high grade and low grade tumours.
\end{abstract}

Keywords: Bladder Neoplasms, Ki 67, Mitotic Count, Proliferative Markers.

\section{Introduction}

Bladder cancer is one of the common neoplasm of lower urinary tract in the present era, which has its own significant morbidity and mortality rates in the society. Hence, it has a great impact on the economic burden of health care systems. Treatment of bladder cancer is very much costlier. Parameters like tumour grading, size, staging alone have minimal role to specify progression, disease recurrence and treatment response. Till now, there are no prognostic markers identified for bladder neoplasms apart from grading and staging.

Many TURBT biopsies do not include muscle in the specimen provided, so invasion cannot be predicted histopathologically ${ }^{(2)}$. In such cases, markers which can detect invasion and confirm higher grade of the tumor will serve as the best guide for patient treatment and survival. Cell proliferation is recently evaluated for biological aggressiveness of bladder tumours. Mitotic count is a most appropriate marker to identify proliferative status in a cancer ${ }^{(3)}$. In those days, proliferation of tumour cells are difficult to measure, though they are clinically significant. Bladder tumours grow rapidly and have doubling time around 70days and hence recurrence occurs very rapidly.
Ki 67 is a monoclonal antibody developed against Hodgkins disease derived cell line L428 and it is seem to interact with nucleus expressed in cells that undergo proliferation. Several authors have published Ki 67 indices in wide range of human tumours like Non Hodgkins lymphoma, prostate, breast and lung carcinomas. It seems to co-relate with aggressive nature of these tumours ${ }^{(4,5)}$.Ki 67 being a nuclear protein that is expressed by cells undergoing proliferation, it can be identified immunohistochemically. ${ }^{(6-9)}$ Due to availability of effective chemotherapy for bladder tumours, there is a great need for accurate prognostic and predictive markers. Therefore, they will help in selecting appropriate patients who are suitable for early intervention and spares those patients from morbidity due to adjuvant therapies. Our study is aimed at considering the use of $\mathrm{Ki}$ 67 staining in urothelial neoplasms of bladder and their correlation with tumour grading and staging.

\section{Materials and Methods}

This is a Prospective study undertaken in the Department of Pathology for a period of 1 year. All TURBT specimens with malignant histology are included in the study. 30 TURBT specimens were subjected into study and were 
evaluated further. Two sections are taken for each case. One for immunohistochemistry and other for routine hematoxyline and eosin staining. Sections for IHC were cut at four micron thickness on to coated slides which are then incubated at 58 degree celcius overnight. Initial sections were stained with hematoxylin and eosin stain.

IHC was done by Two step indirect technique by using ki 67 antibody. Lymph nodes are used as positive control. Antigen retrieval was done by placing slides in microwave in Tris EDTA buffer solution at $\mathrm{Ph}$ 9. DAB was used as a chromogen and hematoxylin used as a counter stain.

The entire sections in a slide is examined under high power objective for the presence of positive immunoreactivity. Tumour cells are read positive if there is golden brown nuclear staining of the neoplastic cells. Semiquantitative scoring was carried out. The intensity of $\mathrm{Ki} 67$ nuclear staining is assessed and graded according to Adams et $\mathrm{al}, 2000^{(11)}$ as depicted in table 1.Then ki 67 grading and histopathological grading is compared and statistically evaluated.

For Ki 67 labelling index each slide is examined under high power and hot spot is selected. Hot spot is an area with highest immunostained nuclei. 1000 tumour cell nuclei is counted and the labelling index is expressed as percentage of positive cells. Caution should be expressed while interpreting $\mathrm{Ki} 67$ positivity as any proliferating cell such as lymphocytes can be positive. Hence hot spot should always be selected after meticulous comparision with routine H\&E sections. All data are analysed with a statistical software package (SPSS, version 16.0 for windows).

\section{Result}

The study was performed on 30 cases of bladder urothelial carcinomas. Male to female ratio is 5:1. The mean age for males is $67 \mathrm{yrs}$ and for females is $47 \mathrm{yrs}$. Males $>60$ years constituting $63 \%$ is the predominant population having bladder neoplasms in our study. Initial clinical presentation in most of the patients is painless hematuria $(77 \%)$. Other symptoms are dysuria (13\%) and urgency (10\%).

Histopathologic grading and staging done and they are distributed as depicted in table no.2.Immunohistochemical analysis with ki 67 was studied and correlated with clinical and morphological parameters.

In present study, high grade tumours have over expression of Ki 67 and graded as 3+ and 4+.Low grade tumours have low expression and have grades $1+$ and $2+$.As depicted in fig 4 , out of 30 cases, $40 \%$ (12 cases) show $4+$ and $27 \%$ show $(8$ cases $) 3+$,both being high grade tumors. Whereas,
$27 \%$ ( 8 cases) have $1+$ and $7 \%$ ( 2 cases) have $2+$, both being low grade tumours.

Out of 30 cases, Ta stage tumors which are non invasive tumors have very low expression of Ki 67 compared to T1 and T2 stage which are invasive. Out of 5 Ta stage tumors 4 cases are $1+$. Out of $6 \mathrm{~T} 1$ tumors 4 cases are $3+$ and 2 cases are $4+$.Out of $19 \mathrm{~T} 2$ stage tumors 9 are $4+, 4$ are $3+, 2$ are $2+$ and 4 are $1+$ as depicted in fig 6 . all 5 cases which had lymphovascular invasion had higher Ki 67 score that is 4+. Statistical analysis showed highly significant between Ki 67 proliferation index, tumour grade $(\mathrm{p}<0.001)$ and tumour stage $(\mathrm{p}<0.001)$.

In our study out of 20 smokers, 4 cases (13\%) were low grade and 16 cases $(53 \%)$ are high grade. Among non smokers, 6 cases (20\%) were low grade and 4cases (13\%) are high grade. Chi square analysis of variables is found to be statistically significant i.e. $p$ value is $<0.05$.

\section{Discussion}

Bladder cancers are emerging as commonest cancer worldwide, whose incidence is rising recently due to habits of tobacco usage and occupational exposure. Many biomolecular pathways are involved in the occurrence of urothelial neoplasms. Around $50-70 \%$ of patients who are muscle-invasive will eventually, may develop metastasis or recurrence after treatment. Annual death rate due to bladder cancer is 12,000. Even though pathologic staging and grading is an important predictor of tumour progression but its value in determinating clinical outcome is very limited. Hence, an independent predictor will enable us to identify individuals who are at increased risk and may benefit from chemotherapy ${ }^{(14)}$.

Uncontrolled cell proliferation is considered to be an important step in tumorigenesis. Ki 67, a nuclear protein is a marker of cell proliferation which is present during G1, S, G2 and M phase of cell cycle. Ki 67 can be detected immunohistochemically in formalin fixed, paraffin embedded tissues. Ki 67 predicts clinical outcome in several other tumours like breast, lung, cervix and prostate tumors. More recently, many investigators have found $\mathrm{Ki}$ 67 as an independent predictor of clinical outcome and response to therapy in bladder cancers.

Transitional cell carcinoma is the commonest variant which accounts for $90 \%$ of bladder neoplasms in the literature. In our study, all patients (100\%) had TCC. Age and gender wise distribution of bladder neoplasm's are shown in table 1. Only $33 \%$ ( 10 out of 30$)$ of the patients are $<60$ years and $67 \%(20$ out of 30$)$ are $>60$ years. Only one female patient had lowest age of presentation i.e. at 28 years. Among 30 patients $83 \%(25)$ are males and $17 \%(5)$ are females. 
Table:1

\begin{tabular}{|c|c|}
\hline Ki 67 nuclear staining & grade \\
\hline$<10 \%$ positivity & $1+$ \\
\hline$<25 \%$ positivity & $2+$ \\
\hline $25-50 \%$ positivity & $3+$ \\
\hline$>50 \%$ positivity & $4+$ \\
\hline
\end{tabular}

Table 2: Clinicopathological Parameters.

\begin{tabular}{|c|c|c|c|}
\hline \multicolumn{2}{|c|}{ Clinicopathological parameters } & No of cases & 33 \\
\hline AGE & $<60$ YRS & 10 & 67 \\
\hline SEX & $>60 Y R S$ & 20 & 83 \\
\hline & MALE & 25 & 17 \\
\hline SMOKING HISTORY & FEMALE & 5 & 80 \\
\hline & SMOKERS & 20 & 20 \\
\hline MUSCLE IN TURBT & NON SMOKERS & 10 & 70 \\
\hline MUSCLE INVASION & INCLUDED & 21 & 30 \\
\hline NUMOUR GRADE & PRESENT & 9 & 63 \\
\hline & ABSENT & 19 & 27 \\
\hline & LOW & 11 & 33 \\
\hline & HIGH & 20 & 67 \\
\hline & Ta & 5 & 17 \\
\hline
\end{tabular}

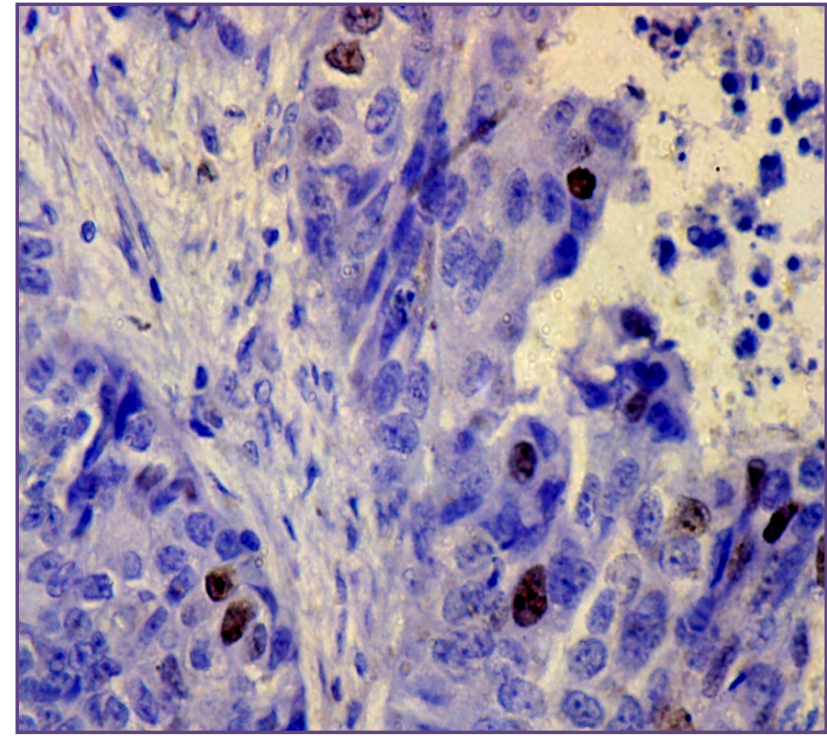

Fig. 1: IHC : KI 67 showing nuclear positivity with score $1+(40 X)$.

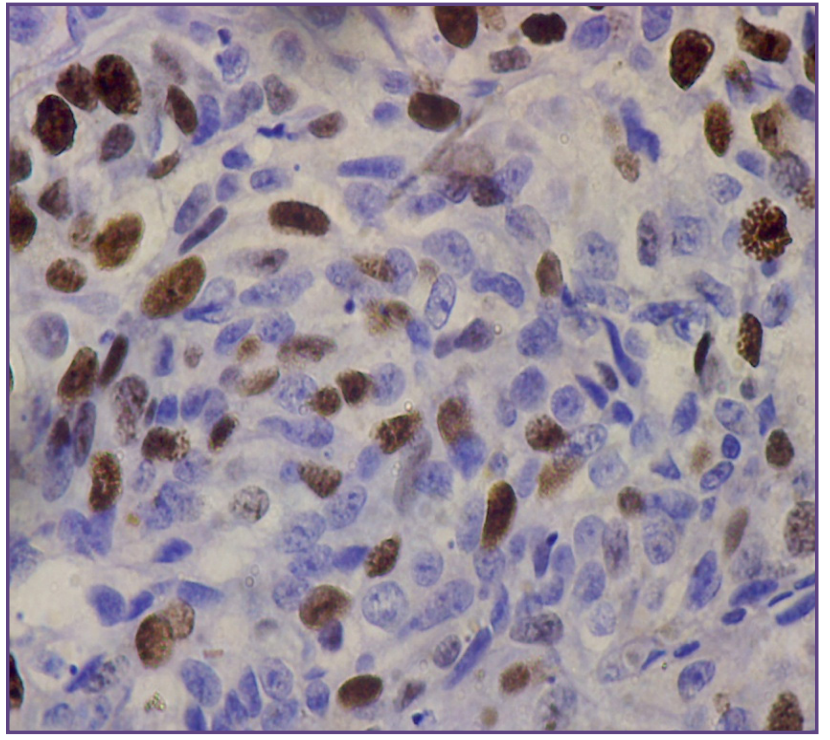

Fig. 2: IHC-KI 67 score $2+(40 \mathrm{X})$. 


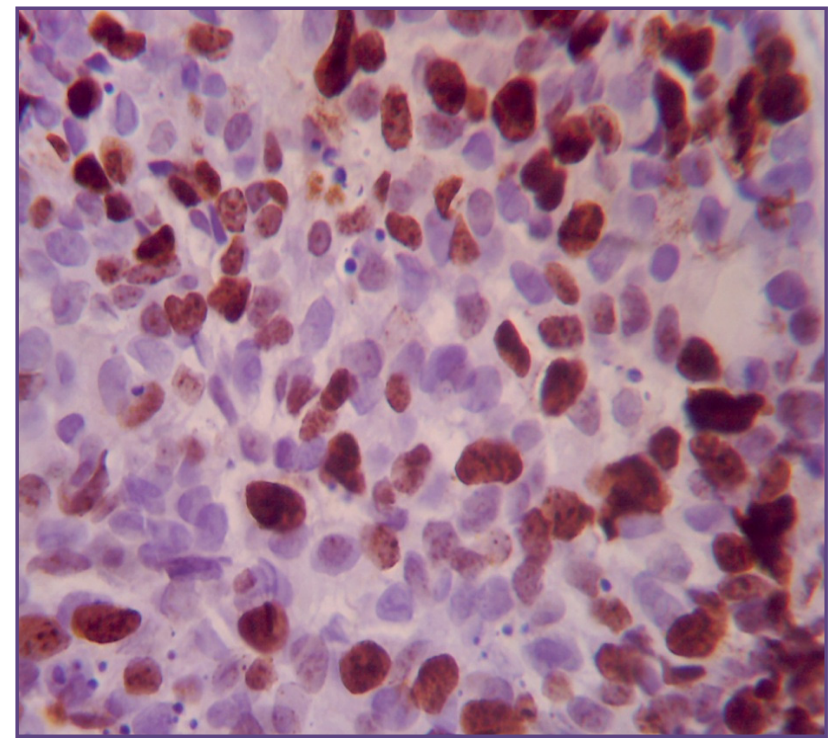

Fig. 3: IHC:KI 67 score $3+(40 X)$.

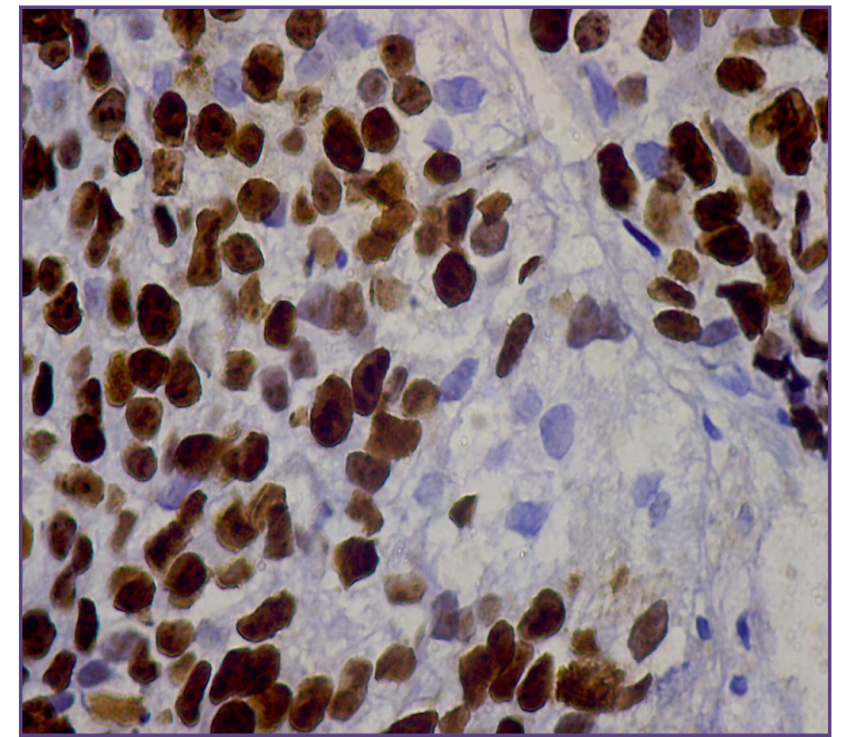

Fig. 4: IHC:Ki 67 score 4+(40X).

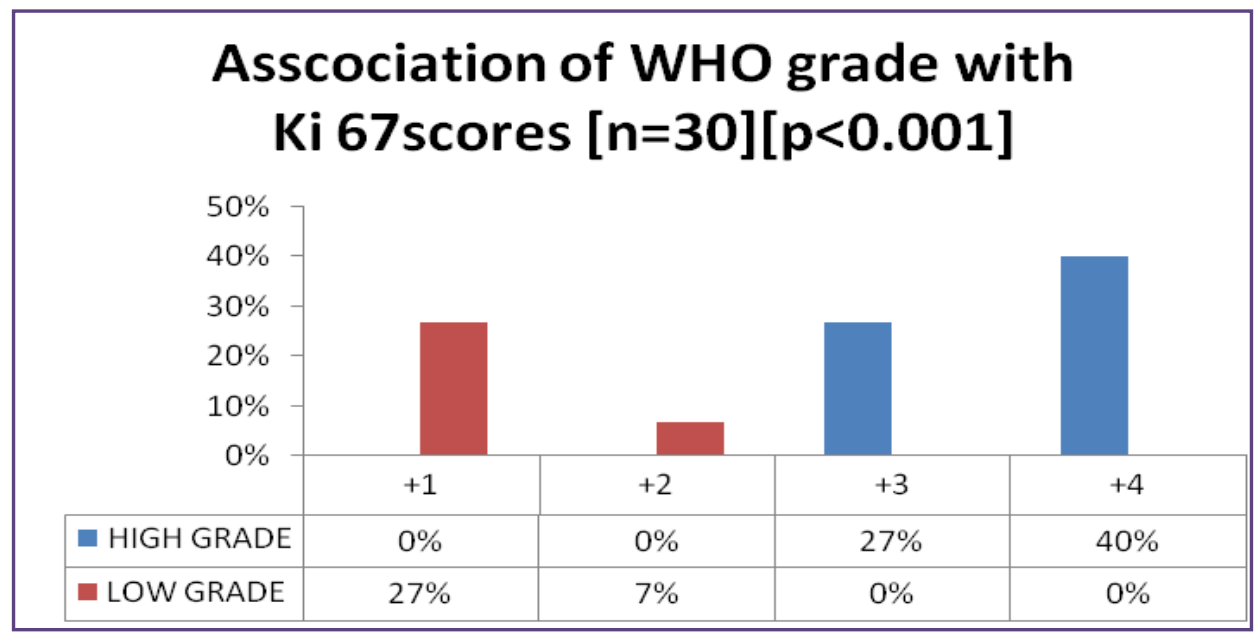

Fig. 5: Ki 67 scoring and WHO grade.

\begin{tabular}{|c|c|c|c|c|}
\hline \multicolumn{5}{|c|}{$\begin{array}{l}\text { Asscociation of Staging with } \\
\text { Ki } 67 \text { scores }[n=30][p<0.05]\end{array}$} \\
\hline \multirow[t]{2}{*}{$\begin{array}{r}40 \% \\
30 \% \\
20 \% \\
10 \% \\
0 \%\end{array}$} & & & & \\
\hline & +1 & +2 & +3 & +4 \\
\hline - Ta (stage Oa) & $13 \%$ & $0 \%$ & $0 \%$ & $3 \%$ \\
\hline - T1 (stage 1) & $0 \%$ & $0 \%$ & $13 \%$ & $7 \%$ \\
\hline T2 (stage 1 ) & $13 \%$ & $7 \%$ & $13 \%$ & $30 \%$ \\
\hline
\end{tabular}

Fig. 6: KI 67 score \& staging. 
The mean age of patients was $64+4.1$ years (range $28-85$ years) and male to female ratio is 5:1.This is in accordance with study conducted by Paraggupta et.al (2009) in which mean age was $60.2+4.4$ and male to female ratio is $8.6: 1^{(20)}$. Similar studies conducted by AlaaS. Jumma(2011) and M. Enache et al(2012) showed mean age of presentation to be $60 \mathrm{yrs}$ and male to female ratio ranges from $3: 1$ to $5: 1^{(7,9)}$.

In our study, $77 \%$ (23 out of 30 ) of the patients presented with painless hematuria. Their increased incidence may be due to lack of screening of microscopic hematuria. Remaining 13\% (4 out of 30 ) presented with dysuria and $10 \%$ (3 out of 30 ) with urgency. This is similar to studies conducted by Paraggupta et.al (2009) in which 97\% of patients presented with painless hematuria ${ }^{(20,12)}$.

Patients were subjected to cystoscopy and then location, number and nature of tumour were recorded. Then transurethral resection of bladder cancer (TURBT) was performed. In the received thirty biopsy specimens around $30 \%$ (9 out of 30 ) did not have muscularispropriae in them (chart-5).This is similar to Paraggupta et.al((2009) in which $34.5 \%$ of the patients did not have detrusor muscle in their TURBT specimens ${ }^{20}$.Also this is concordance with Maruniak et al(2002)study, which states muscularispropria was absent in $40-50 \%$ of TURBT specimens. This leads to understaging in many patients ${ }^{(2)}$.

Total of $63 \%$ showed muscle invasive disease at time of presentation. Remaining $27 \%$ of the patients are nonmuscle invasive. Since, patients arrive at the later stage to the hospital, many are found to be muscle invasive. This is similar to study conducted by Alaa S Jumaa(2011) in which $57 \%$ of patients constituted muscle invasive disease and in study conducted by M.Enache et.al(2012) $52 \%$ constituted T2-T4 disease $^{(9)}$.

Cigarette smoking is one of the risk factor which causes increased incidence of bladder tumours, at least 4-5 times than the normal population in both males and females. In our study, history of smoking is more in males $(66 \%)$ and all females are non smokers. In the study by paraggupta et.al (2009) incidence among male smokers ( $78 \%$ vs $22 \%$ ) are higher compared to females ${ }^{(20)}$.

In our study 20 cases $(67 \%)$ are high grade tumors and $10(33 \%)$ cases are low grade tumors. All 30 cases were subjected to $\mathrm{Ki} 67$ immunostaining and was graded according to Adams et al (2000)from $1+$ to $4+^{(11)}$. The results obtained are, $40 \%$ ( 8 out of 20 ) of high grade tumors are $3+$ and $60 \%(12$ out of 20$)$ of high grade tumors are $4+$.Among low grade tumors $80 \%(8$ out of 10$)$ are graded as $1+$ and remaining $20 \%(2$ out of 10$)$ have score of $2+$.From these results we can say that all high grade tumors had higher proliferation rate and had score of $3+$ and $4+$, whereas low grade tumors have score of $1+$ and $2+($ fig 5 ). Thus tumor grade and Ki 67 scoring correlated well in our study with $\mathrm{p}$ value being highly significant $(\mathrm{p}:<0.001)$. This is similar to study conducted by Alaa S. Jumaa (2011) in which 33\%(7 out of 21) of high grade cases showed 3+ and 66\%(14 out of 21 ) of cases showed $4+$. Whereas in low grade tumours, $75 \%$ (21 out of 28 ) of cases showed $1+$ and $25 \%$ ( 7 out of 28 ) showed 2+.This study also concluded that Ki 67 scores correlates with tumour grading(7).

Another study conducted by M. Enache et.al(2012) states that $\mathrm{Ki} 67$ index was positive in $72 \%$ ( 32 cases) with maximum in moderately and poorly differentiated grades and $p$ value was statistically significant $(\mathrm{p}<0.001)$. Another study by Goyal et.al(2014) and Vitalymargulis et.al (2009),states that Ki 67 scoring have stronger corelationship with tumor grading and hence it may serve as a reliable indicator of clinical outcome ${ }^{(1,69)}$.

In the present study, we can make out that Ta stage tumors have very low Ki 67 expression (4 out of 5) and was given score $1+$.Whereas $\mathrm{T} 1$ and $\mathrm{T} 2$ stage tumors have high expression of Ki 67 and was graded as $3+$ and 4+.Thereby, stage correlated well with Ki 67 scoring and it is statistically significant $(\mathrm{p}<0.05)($ fig 6$)$.Similar studies conducted by Alaa S Jumaa (2011) states that T2 stage tumours have higher expression of Ki 67 with score of 4+ and Ta ,T1 tumours have lower expression with score $1+\left({ }^{(7)}\right.$. These results were also consistent with studies conducted by Nakopoulol et al(1998),Lei C.X. et al(2010), and Pich A et al (2002)which states that Ki 67 expression correlates with grade and stage of tumor. Hence Ki 67 serves as a prognostic marker in indicating biologic aggressiveness of the disease. ${ }^{(3,14,15)}$

In the present study, 5 out of 30 cases (16\%) showed lympho vascular invasion. All these 5 cases had Ki 67 scoring of $4+$ and they are found statistically significant $(\mathrm{p}<0.05)$. This is in concordance with Vitaly Margulis et al(2006) in which Ki 67 over expression was associated significantly with lymphovascularinvasion. Thus, Ki 67 helps in identifying patients with worst clinical outcome ${ }^{(6)}$.

In the present study, in patients less than age 60 years there is equal distribution of low and high grade tumours $(50 \%$ Vs $50 \%$ ). whereas as age advances $>60 \mathrm{yrs}$, there is increased incidence of high grade tumours ( $25 \%$ Vs $75 \%$ ). This is similar to study by paraggupta et al(2009) in which 
younger individuals present with lower grade and stage of tumours compared to elderly patients ${ }^{(20)}$. This is actually contrast to common nature of malignancy in which biological behavior of malignancy is very aggressive in younger individuals.

In our series, males have increased incidence of high grade tumours compared to females and also smokers had higher occurrence of high grade tumours compared to non smokers. Possible explanations are, males have increased risk of exposure to many environmental and dietary exposures and also smoking may increase additional mutations in urothelium, thus causing high grade disease.

\section{Conclusion}

These observations emphasize the use of Ki 67, in addition to tumour grade and stage to predict the clinical outcome of patients and thereby helps in identifying high risk patients. These patients are frequently followed up and may benefit by additional adjuvant therapies. Clinical importance of this marker prompts multi institutional prospective studies with large sample size. Awareness among public is needed as they ignore hematuria symptoms and presents to the clinicians at advanced stage of the disease. Reason behind aggressive tumors in elderly is not known and hence research should be carried out furthur.

\section{Acknowledgements}

We would like to thank our colleagues and technicians for their expert and technical help in carrying out the study.

\section{Reference}

1. Surbhi G,1 Usha RS,1 Sonal S,1 Navneet K 2. Correlation of Mitotic Indices, AgNor Count, Ki-67 and Bcl-2 with Grade and Stage in Papillary Urothelial Bladder Cancer, uro J 2014:11:1239-47

2. Maruniak NA, Takezawa K, Murphy WM. Accurate pathologic staging of urothelial neoplasm requires better cystoscopic sampling. J Urol 2002;167:2404-7.

3. Nakopoulou L, Vourlakou C, Zervas A, Tzonou A, Gakiopoulou H, Dimopoulos MA. The prevalence of bcl2, p53, and Ki-67 immuno-reactivity in transitional cell bladder carcinomas and their clinicopathologic correlates. Hum Pathol 1998;29:146 -54

4. Li R, Heydon K, Hammond ME, Grignon DJ, Roach M 3rd, Wolkov HB, Sandler HM, Shipley WU, Pollack A, Ki67 staining index predicts distant metastasis and survival in locally advanced prostate cancer treated with radiotherapy: an analysis of patients in radiation therapy oncology groupprotocol 86-10, Clin Cancer Res, 2004, 10(12 Pt 1):4118-4124.

5. Gimotty PA, Van Belle P, Elder DE, Murry T, Montone KT, Xu X, Hotz S, Raines S, Ming ME, Wahl P, Guerry
D, Biologic and prognostic significance of dermal Ki67 expression, mitoses, and tumorigenicity in thin invasive cutaneous melanoma, J Clin Oncol, 2005, 23(31):8048-8056

6. MargulisV, Shariat1 S.F, Ashfaq R, Sagalowsky A, and Lotan Y. Ki-67 Is an Independent Predictor of Bladder Cancer Outcome in Patients Treated with Radical Cystectomy for Organ-Confined Disease. doi: 10.1158/1078-0432.CCR-061472 Clin Cancer Res December 15, 2006 12; 7369

7. Alaa S. Jumma et.al. the assessment of proliferative activity in transitional cell carcinoma of the urinary bladder by using ki 67 overexpression and its effects on prognosis of the tumor in correlation to prognostic markers (staging and grading); immunohistochemical study. kufa me jour 2011:vol 14.no.2

8. Ding W, Gou Y, Sun C, Xia G, Wang H, Chen Z, Tan J, Xu $\mathrm{K}$, Qiang D,KI 67 IS AN INDEPENDENT predictor in non muscle invasive bladder cancer(NMIBC);Combination of EORTC risk scores and ki 67 expression could improve the risk stratification of NMIBC, Urol onco,2014 Jan ;32(1):42.

9. Enache M, Cristiana S, Luana CL. KI 67 and BCL2 immunoexpression in primitive urothelial bladder carcinoma. Rom J Morphol Embryol 2012,53(3):521-525.

10. Maximillian burger et.al. epidemiology and risk factors of urothelial bladder cancer. European urology 2013:234-241.

11. Adam J, Carder PJ, Downey S, Fobes MA, MacLennan K, Allgar V, Kaufman S, Hallam S, Bicknell R, Walker JJ, Cairnduff F, Selby PJ, Perren TJ, Lansdown M, and Banks RF:VEGF in breast cancer: comparison of plasma , serum and tissue VEGF and micro vessels density and effects of tamoxifen. Cancer research June 1, 2000:66:2898-2905

12. Michael N, Athanasios P, Maria-EK, Stavros T, Ioannis V, Georgios S, Georgios A, and Ploutarchos A. Urothelial Carcinoma of the Urinary Bladder in Young Adults: Presentation, Clinical behavior and Outcome. Advances in Urology, Volume 2011, Article ID 480738.

13. Augustine A, Hebert JR, Kabat GC, Wydner EL. Bladder cancer in relation to cigarette smoking. Cancer Res. 1988;48:4405-8.

14. Pich A, Chiusa L, Formiconi A, Galliano D, Bortolin P, Navone R (2001). Biologic differences between non invasive papillary urothelial neoplasms of low malignant potential and low-grade (grade1) papillary carcinomas of the bladder. Am J Surg Pathol 25: 1528-15334

15. Lei C.X, Bing-xun L, Hai-lin Ch., Qi-guang Ch, Zhe Z, Jian-bin B, Chui-ze K. Expression and Significance of Fascin and Ki-67 Proteins in BladderCarcinoma. Journal of China Medical University 2010-02 [DOI] $\square$ CNKI:SUN:ZG YK.0.2010-02-017

16. Ro JY, Ayala AG, El-Naggar A. Muscularis mucosa of urinary bladder: importance for staging and treatment. Am J Surg Pathol 1987;11:668-673.

17. Philip AT, Amin MB, Tamboli P, et al. Intravesical adipose tissue. Quantitative study of its presence and location with 
implications for therapy and prognosis. Am J Surg Pathol 2000;24:1286-1290.

18. Moore KL. Clinically oriented anatomy, 2nd ed. Baltimore: Williams \& Wilkins, 1985:265.

19. Korkolopoulou P, Christodoulou P, Kapralos P, Exarchakos M, Bisbiroula A,Hadjiyannakis M, Georgountzos C,
Thomas-Tsagli E (1997). The role of p53, MDM2 and c-erb B-2 oncoproteins, epidermal growth factor receptor and proliferation markers in the prognosis of urinary bladder cancer. Pathol Res Pract 193: 767-775.

20. Parag $G$ et.al. Impact of age and gender on the clinicopathological characteristics of bladder cancer. Indian j urol.2009:207-210.

\section{*Corresponding author:}

Dr. Megala .C, Assistant Professor, Department of Pathology, Vinayaka Missions Kirupananda Variyar Medical College, Salem-636308, Tamil Nadu, INDIA. Phone: +91 9677448928

Email: drmegala1151989@gmail.com

Financial or other Competing Interests: None. 